\title{
Quantitative analysis based on chest CT classifies common and severe patients with coronavirus disease 2019 pneumonia in Wuhan, China
}

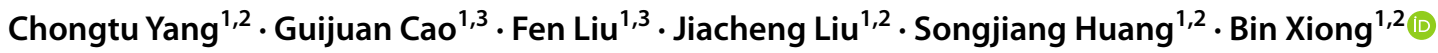

Received: 5 January 2021 / Revised: 19 February 2021 / Accepted: 2 March 2021 / Published online: 8 April 2021

(c) The Author(s), under exclusive licence to Springer Nature Singapore Pte Ltd. 2021

\begin{abstract}
Objective This study aimed to compare quantifiable radiologic findings and their dynamic change throughout the clinical course of common and severe coronavirus disease 2019 (COVID-19), and to provide valuable evidence for radiologic classification of the two types of this disease.

Methods 112 patients with laboratory-confirmed COVID-19 were retrospectively analyzed. Volumetric percentage of infection and density of the lung were measured by a computer-aided software. Clinical parameters were recorded to reflect disease progression. Baseline data and dynamic change were compared between two groups and a decision-tree algorithm was developed to determine the cut-off value for classification.

Results 93 patients were finally included and were divided into common group $(n=76)$ and severe group $(n=17)$ based on current criteria. Compared with common patients, severe patients experienced shorter advanced stage, peak time and plateau, but longer absorption stage. The dynamic change of volume and density coincided with the clinical course. The interquartile range of volumetric percentage of the two groups were 1.0-7.2\% and 11.4-31.2\%, respectively. Baseline volumetric percentage of infection was significantly higher in severe group, and the cut-off value of it was $10.10 \%$.

Conclusions Volumetric percentage between severe and common patients was significantly different. Because serial CT scans are systemically performed in patients with COVID-19 pneumonia, this quantitative analysis can simultaneously provide valuable information for physicians to evaluate their clinical course and classify common and severe patients accurately.
\end{abstract}

Keywords Coronavirus disease $2019 \cdot$ Multidetector computed tomography · Artificial intelligence $\cdot$ Numerical analysis · Computer-assisted $\cdot$ Decision trees

\section{Abbreviations}

COVID-19 Coronavirus disease 2019

CAD Computer-aided diagnosis

SARS-CoV-2 Severe acute respiratory syndrome coronavirus 2

IQR Interquartile range
RT-PCR

PACS

$\mathrm{SaCO}_{2}$

GGO
Reverse transcription polymerase chain reaction

Picture archiving and communication systems

Oxygen saturation

Ground glass opacity

Guijuan Cao and Chongtu Yang contributed equally.

\author{
Bin Xiong \\ herr_xiong@126.com \\ Chongtu Yang \\ henrys1011@163.com \\ Guijuan Cao \\ cao334647288@163.com \\ Fen Liu \\ fen13667280651@163.com \\ Jiacheng Liu \\ jiacheng6jc@163.com
}

Songjiang Huang

hsjhzkjdx@163.com

1 Department of Radiology, Union Hospital, Tongji Medical College, Huazhong University of Science and Technology, Jiefang Avenue \#1277, Wuhan 430022, China

2 Hubei Province Key Laboratory of Molecular Imaging, Wuhan 430022, China

3 Department of Radiology, Wuhan Central Hospital, Wuhan, Hubei, China 


\section{Introduction}

Since early December 2019, a cluster of pneumonia cases of unknown aetiology has been reported in Wuhan China [1-3], and the disease has spread rapidly to other province of China. The pathogen was then named severe acute respiratory syndrome coronavirus 2 (SARS-CoV-2) by WHO and the disease caused by it was termed the coronavirus disease 2019 (COVID-19) [4, 5].

Patients with COVID-19 pneumonia have a clinical spectrum ranging from mild to critically ill $[6,7]$. Thus, early classification and identification of patients who have high risk of poor prognosis may help them benefit from more aggressive treatment. Baseline data, including laboratory tests, pulmonary function measures and computed tomography (CT) findings, are helpful in classifying patients into different types and thus predicting disease progression and patients' prognosis. Until now, several clinical parameters have been applied to define the severity of COVID-19 pneumonia [8], but accurate radiological assessment and classification criteria remain elusive due to individual physiological variability and the semiquantitative nature of $\mathrm{CT}$ which may be evaluated inconsistently between radiologists.

Computer-aided diagnosis (CAD) refers to image analysis tools developed to assist image reading. By automatically detecting and quantifying abnormalities on CT, it can objectively provide an accurate and reproducible evaluation of disease $[9,10]$. We designed this retrospective study aiming to: (1) access the accuracy of quantifiable radiologic findings in reflecting the clinical course of patients with common and severe COVID-19 pneumonia and (2) provide valuable evidence for clinical and radiologic classification of the two types of this disease by developing a decision-tree algorithm.

\section{Materials and methods}

\section{Study design}

This observational study was conducted at Wuhan Central Hospital and Wuhan Union Hospital, both of which were assigned by the government to treat COVID-19 patients. The study protocol was complied with the principles of the Declaration of Helsinki and was approved by the institutional review board of both centers. Informed consent was waived, because the data were anonymous.

\section{Patients and data collection}

Between January 2020 and February 2020, all consecutive patients with confirmed diagnosis of COVID-19 pneumonia admitted to Wuhan Central Hospital and underwent serial chest CT scans were screened for participation in the study. Exclusion criteria were: (1) patients received less than three times of chest CT scans during hospitalization; (2) abnormalities were not found on CT; (3) pneumothorax or other lung diseases which made volume unable to be measured accurately; and (4) acute severe co-existing diseases, including infection and acute coronary syndrome. SARS-CoV-2 infection was diagnosed at admission or during hospitalization with the reverse transcription polymerase chain reaction (RT-PCR) or gene sequencing. Details of laboratory confirmation processes have been described previously [11].

Data were gathered from electronic medical records in the context of standard practice. Information regarding epidemiological characteristics, clinical symptoms and signs, co-existing disease, laboratory parameters, chest CT scans, treatment and outcomes were recorded with data collection form from admission to discharge. The date of disease onset was defined as the date typical symptoms occurred. Oxygen saturation $\left(\mathrm{SaO}_{2}\right)$ was monitored by fingertip oximeter daily and nightly or by blood gas analysis (BGA), and was used as a main parameter to reflect patient's conditions. Data were censored at the end of the study.

Patients without exclusion criteria were divided into two groups (the common group and the severe group) according to the latest guideline of Diagnosis and Treatment of Pneumonitis Caused by 2019-nCoV (trial sixth version), which classified patients into four types: mild, common, severe and critically ill. Common type was defined as patients with fever, respiratory symptoms and radiological features of pneumonia, while type of severe was diagnosed if patients met one of the following criteria at admission: (1) severe respiratory distress (respiratory rate $[R R]>30$ breaths/ min); (2) oxygenation index $\left(\mathrm{PaO}_{2} / \mathrm{FiO}_{2}\right) \leq 300 \mathrm{mmHg}$ $(1 \mathrm{mmHg}=0.133 \mathrm{kPa})$; and (3) $\mathrm{SaO}_{2} \leq 93 \%$ at rest. Identification of patients' type was achieved by analyzing clinical parameters at admission and reviewing medical logs. Patients who were diagnosed as common at admission but met one of the above criteria during hospitalization, were defined as common to severe, and their CT data before turning into severe were classified into common group, while data after that were classified into severe group.

\section{Chest CT protocol and volumetric quantification}

Ninety-three patients underwent a total of 371 chest CT scans during hospitalization, with an average of 3.99 times for each patient. The interval between the disease onset and the first CT scan was 4.0 days (IQR 2.0-7.5), and the interval between two serial scans was 6.0 days (IQR 5.0-8.0).

Chest CT scans were performed during inspiration in the supine position using one of the following MDCT scanners (Philips Brilliance Core128; Siemens Healthineers, 
Germany; United Imaging, China). All CT scans were non-contrast with the following parameters: collimation, $64 \times 0.6 \mathrm{~mm}$; tube voltage, $120 \mathrm{kV}$, with automatic tube current modulation. Images were reconstructed with a slice thickness of 0.625 or $1.250 \mathrm{~mm}$ and were transferred to the workstation to receive further reconstruction.

Two investigators searched for patients' CT images from the picture archiving and communication systems (PACS) and used the software "Pneumonia Doc (Version 1.17.0, ShuKun Technology)" to perform quantitative assessment. "Pneumonia Doc" uses a deep learning algorithm based on $3 \mathrm{~d}$ U-NET neural networks and is specially developed to help radiologists detect and evaluate COVID-19 pneumonia. Data measurement mainly included three steps: segmentation, classification and quantification. First, lung parenchyma was automatically segmented into five regions (left upper, left lower, right upper, right middle and right lower lobe). Then the density was measured and slice images were classified into seven types of tissues automatically: background, lung field, effusion, ground-glass opacity (GGO), pulmonary fibrosis, interstitial thickening and consolidation. Afterwards, two types of lung tissues were identified based on their characteristics (including GGO and consolidation): infected lesions and normal area (Fig. 1). Two researchers checked the segmentation and classification process, who were blinded to the clinical data. If discrepancy existed, three experienced radiologists discussed it and made the final decision. Volumes of both tissues in each lobe were calculated and expressed in milliliters, and total volume was the volumetric sum of each lobe. Percentage of infection of each lobe was defined as the ratio of abnormal volume of each lobe divided by corresponding lobe volume, with total percentage of infection defined as the sum of percentage of infection of each lobe. Besides, density of each lobe and the whole lung was measured and was divided into seven ranges based on their Hounsfield unit $(\mathrm{Hu})$. Volume and density data based on the first and follow-up CT of each patient were recorded for dynamic change analysis.

\section{Statistical analysis}

Quantitative variables were expressed as means and standard deviation (SD) or median and interquartile range (IQR), and compared using Student's $t$ test or Mann-Whitney test. Qualitative variables were presented as frequencies and percentages, and compared by means of $\chi^{2}$ test or two-tailed Fisher's exact test. Linear regression analyses were used to evaluate the correlation between quantitative lesion size and clinical parameters $\left(\mathrm{SaO}_{2}\right.$, leucocyte count, neutrophil count, lymphocyte count and $\mathrm{C}$-reactive protein). To provide accurate and facilitate implementation for radiologic classification, we used a decision-tree algorithm to test whether a specific volumetric percentage could classify COVID-19 patients into common or severe group defined by current criteria. A $P$ value of less than 0.05 was considered statistically significant. Statistical analyses were processed using IBM SPSS (version 25.0) and GraphPad Prism (version 9.0) was used to draw pictures.
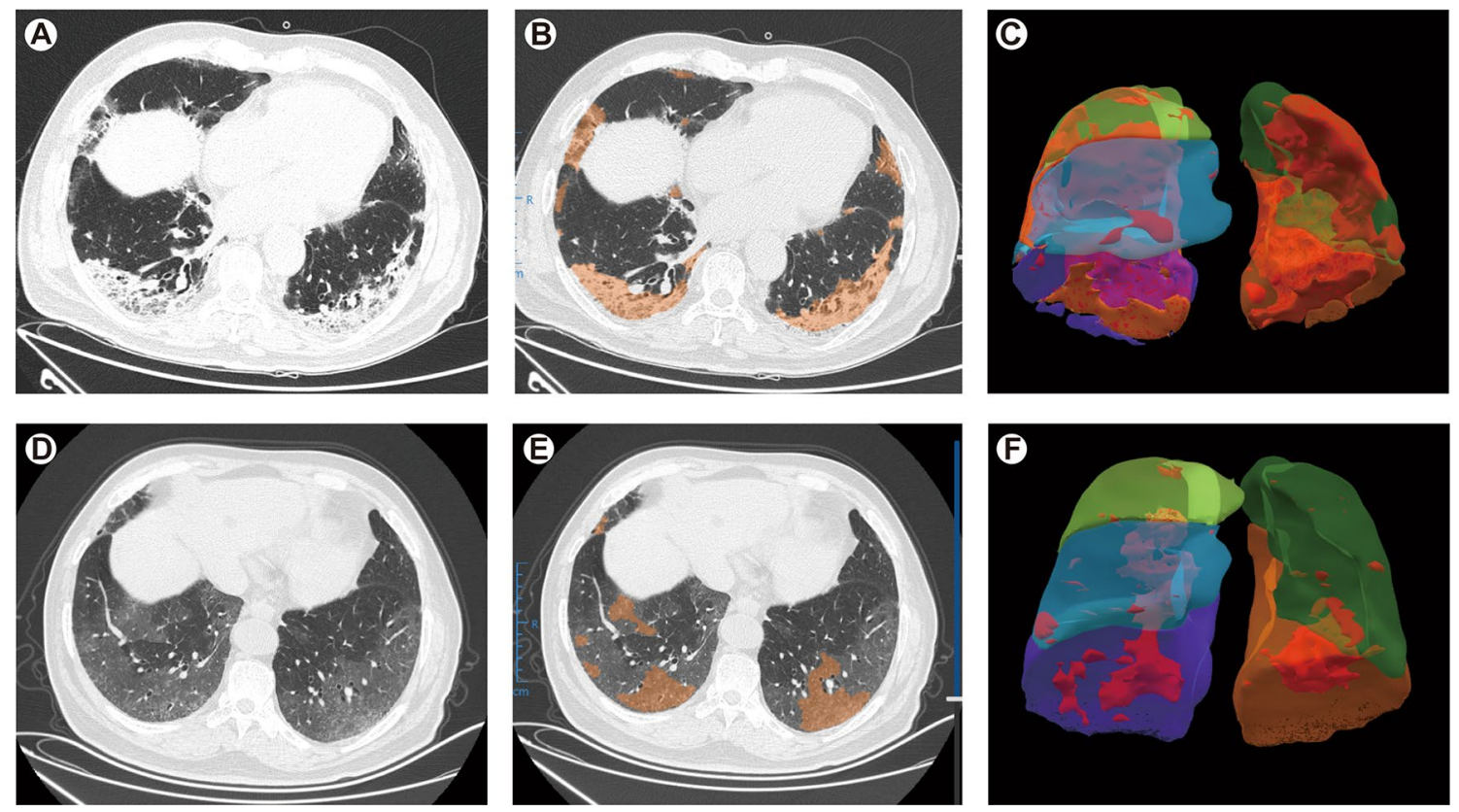

Fig. 1 Identification of abnormalities by the CAD software. a-f Images from the same person at two time-points. a, $\mathbf{d}$ Original images; $\mathbf{b}, \mathbf{e}$ automatically identified and colored; $\mathbf{c}, \mathbf{f}$ three-dimensional reconstruction with each lobe has its color and orange represents lesions 


\section{Results}

\section{Study population}

A total of 112 patients with laboratory-confirmed COVID-19 admitted to our center from January 4 to February 17 were retrospectively analyzed. Among them, patients with less than three CT scans $(n=4)$, without any abnormalities on CT $(n=10)$ and with acute coronary syndrome $(n=1)$ were excluded. Besides, 4 patients had no records of $\mathrm{SaO}_{2}$ and clinical classifications at admission and were also excluded. The final analysis included 93 patients with a median age of 57.0 (IQR, 39.0-67.0), of whom 76 (82\%) were defined as common and 17 (18\%) were severe. Among the 17 severe patients, 16 of them had a $\mathrm{SaO}_{2}$ lower than $93 \%, 5 \mathrm{had}$ an oxygenation index lower than $300 \mathrm{mmHg}$. Table 1 summarizes the baseline characteristics of the study population.

\section{Volumetric analysis and dynamic change}

To investigate whether quantitative analysis based on radiological findings of both common and severe patients coincided with their clinical course and with previous studies, the dynamic change of volumetric percentage of infection and density of the lung based on follow-up CT were analyzed in all patients (Fig. 2a). Both two groups experienced four stages: advanced stage, peak time (from disease onset to the peak of total percentage of infection), plateau and absorption stage. Compared with the common group, the peak time in severe group was earlier (approximately 12-9 days, respectively), and the plateau stage was shorter. The median total percentage of infection of all follow-up CT scans were $3.1 \%$ (IQR, 1.0-7.2\%) in common group and $19.4 \%$ (IQR, $11.4-31.2 \%$ ) in severe group.

Eleven patients (14.5\%) in the common group progressed from common at admission to severe during hospitalization, and their dynamic change of percentage of infection was also analyzed (Fig. 2b). The mean time from admission to reaching to severe degree was 13.6 days (6-22 days). In 8 patients (73\%), the interval between clinical diagnosis of severe and severe degree on CT (volumetric percentage reached to the lower limit of interquartile range of the severe group) was within 3 days. Among the 11 patients, 9 discharged and 2 patients were finally died despite using extracorporeal membrane oxygenation (ECMO).

Table 1 Clinical characteristic of the study population

\begin{tabular}{|c|c|c|c|}
\hline & $\begin{array}{l}\text { All patients } \\
(n=93)\end{array}$ & $\begin{array}{l}\text { Common } \\
(n=76)\end{array}$ & $\begin{array}{l}\text { Severe } \\
(n=17)\end{array}$ \\
\hline \multicolumn{4}{|l|}{ Age } \\
\hline Median (IQR)-y & $57.0(39.0-67.0)$ & $53.0(35.0-63.0)$ & $68.0(56.0-72.0)$ \\
\hline$<50$ no. $(\%)$ & $35(37.6)$ & $35(46 \%)$ & $0(0)$ \\
\hline$\geq 50$ no. $(\%)$ & $58(62.4)$ & $41(54 \%)$ & $17(100)$ \\
\hline Male sex no. (\%) & $48(51.6)$ & $40(52.6)$ & $8(47.0)$ \\
\hline \multicolumn{4}{|l|}{ Symptoms at admission no. (\%) } \\
\hline Fever & $74(79.6)$ & $61(80.2)$ & $13(76.4)$ \\
\hline Cough & $58(62.4)$ & $43(56.5)$ & $15(88.2)$ \\
\hline Sputum & $32(34.4)$ & $24(31.6)$ & $8(47.0)$ \\
\hline Dyspnea & $39(41.9)$ & $28(36.8)$ & $11(64.7)$ \\
\hline \multicolumn{4}{|l|}{ Co-existing disease no. (\%) } \\
\hline COPD & $2(2.1)$ & $1(1.3)$ & $1(5.9)$ \\
\hline Diabetes & $11(11.8)$ & $4(5.2)$ & $7(41.2)$ \\
\hline Hypertension & $19(20.4)$ & $13(17.1)$ & $6(35.3)$ \\
\hline Coronary heart disease & $6(6.4)$ & $5(6.5)$ & $1(5.9)$ \\
\hline Cerebrovascular disease & $1(1.0)$ & $0(0.0)$ & $1(5.9)$ \\
\hline $\begin{array}{l}\text { Time between disease onset and the first CT scan (median, IQR) } \\
\text { (days) }\end{array}$ & $4.0(2.0-7.5)$ & $3.0(1.0-6.0)$ & $7.0(6.0-9.0)$ \\
\hline Mean number of CT scans & 3.98 & 4.02 & 3.82 \\
\hline Time between the two CT scans (median, IQR) (days) & $6.0(5.0-8.0)$ & $6.0(5.0-8.0)$ & $6.0(5.0-9.0)$ \\
\hline \multicolumn{4}{|l|}{ Clinical outcome no. $(\%)$} \\
\hline Discharge & $89(95.7)$ & $74(97.4)$ & $15(88.2)$ \\
\hline Death & $4(4.3)$ & $2(2.6)$ & $2(11.8)$ \\
\hline
\end{tabular}

$I Q R$ interquartile range, $C O P D$ chronic obstructive pulmonary disease 
Fig. 2 Timeline of the percentage of total infection. Figure 1a compares the dynamic change between severe group and common group. Figure 1b illustrates the percentage of total infection in patients diagnosed as common but aggravated to severe during hospitalization
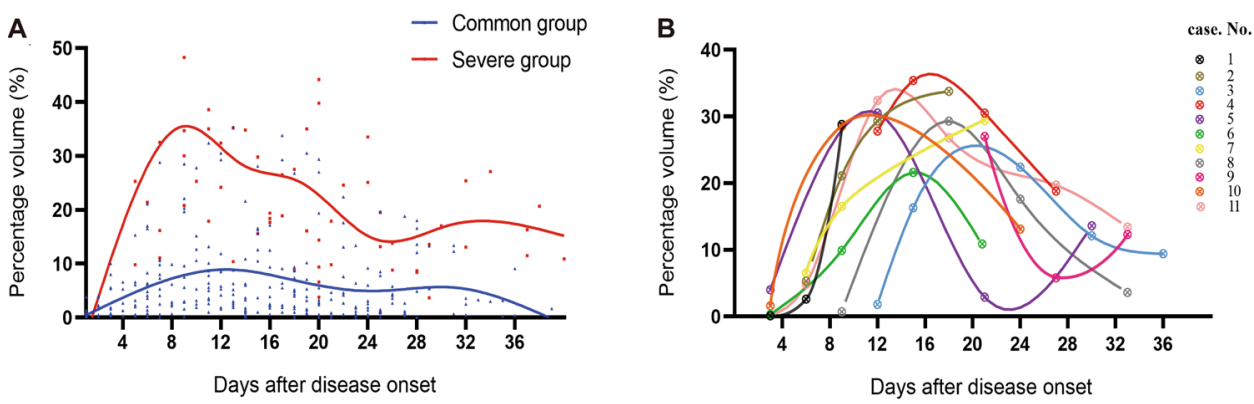

\section{Baseline quantitative data analysis}

Baseline total percentage of infection was significantly lower in the common group than in severe group (2.76 versus $32.18 \%, P<0.001)$. In the common group, the right lower lobe and left lower lobe were the most frequently involved segments, as $18(27.7 \%)$ and $30(46.2 \%)$ patients' main infected lesions located in these two lobes. But in severe group, the most common involved segments were the right lower lobe and right upper lobe, with the infection extent of 10.0\% (IQR 0-16.5\%) and 4\% (IQR 0-19.0\%), respectively. Table 2 demonstrates the baseline volumetric quantification for total lung and for each lobe. Furthermore, Pearson analysis showed slight correlation between total lesion size and the level of neutrophils $(r=0.351, P=0.04)$. However, total lesion size was not correlated with other clinical parameters.

\section{Decision-tree algorithm for classification}

The decision-tree algorithm was developed to determine the cut-off value of common and severe degree. The classifier included was total percentage of infection. Terminal nodes represent the number of patients in each group, and branches represent the cut-off value (Fig. 3a). The cut-off value calculated by the decision-tree was $10.10 \%$, which means patients with total percentage of infection lower than
$10.10 \%$ at admission will be defined as common, otherwise will be defined as severe (Fig. 3b). The sensitivity and specificity of the decision-tree algorithm were 94.7 and $97.3 \%$, respectively.

\section{Discussion}

Since the outbreak of COVID-19 pneumonia, a large amount of studies have evaluated the feasibility and effectiveness of chest CT scans applied for patients with COVID-19 pneumonia. Most of these studies focused on the radiological features of this novel disease [12-17], and the data are sparse with regard to the quantitative $\mathrm{CT}$ analysis. Several studies investigated the association between quantitative CT data and adverse clinical outcomes [18-20], but few related the dynamic change of these data with patients' clinical course. In our study, we compared and quantitatively analyzed the common and severe patients with COVID-19 pneumonia. Our results showed that quantitative analysis based on CT was coincided with the clinical course. The interquartile range of total percentage of infection was $11.4-31.2 \%$ and $1.0-7.2 \%$, respectively. The cut-off value of volumetric percentage of the severe and common degree was $10.10 \%$.

The dynamic change of COVID-19 pneumonia in our study was similar to two recent studies, in which reported
Table 2 Compared baseline radiological data in common versus severe

\begin{tabular}{lllllll}
\hline Volume measurement & \multicolumn{2}{l}{ Common $(n=76)$} & & Severe $(n=17)$ & $P$ value $^{*}$ \\
\cline { 2 - 3 } & Frequency $(\%)$ & Volume (\%) & & Frequency ${ }^{\mathrm{a}}(\%)$ & Volume $(\%)$ & \\
\hline Total lung & NA & $1.5(0.2-5.4)$ & & NA & $33.0(22.5-37.0)$ & $<0.001$ \\
Left upper lobe & 15.4 & $0(0-0.4)$ & & 5.8 & $2.0(0-14.5)$ & 0.003 \\
Left lower lobe & 27.7 & $0.2(0-0.6)$ & & 17.6 & $1.0(0-15.0)$ & 0.008 \\
Right upper lobe & 9.2 & $0(0-0.3)$ & & 35.4 & $4(0-19.0)$ & 0.01 \\
Right middle lobe & 1.5 & $0(0-0.1)$ & & 0.0 & $0(0-0)$ & 0.79 \\
Right lower lobe & 46.2 & $0.2(0-1.5)$ & 41.2 & $10.0(0-16.5)$ & 0.006 \\
\hline
\end{tabular}

NA not applicable

${ }^{a}$ Frequency is calculated as the number of lobes including the main lesion at admission divided by the number of patients (76 in non-severe group and 17 in severe group)

*Nonparametric continuous variables were compared through Mann-Whitney $U$ test for independent samples. $P<0.05$ was considered statistically significant 
A

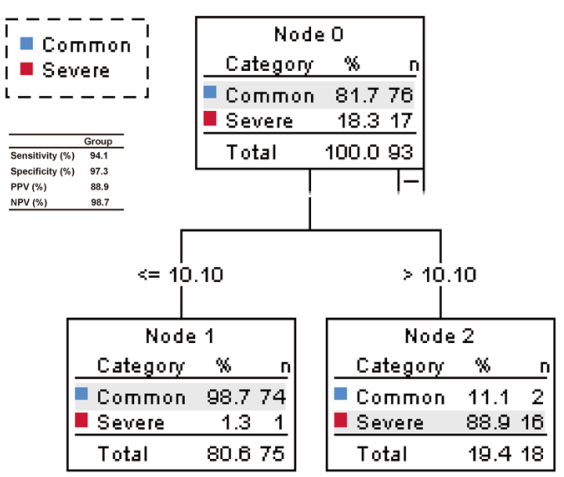

Fig. 3 Decision-tree algorithm and scatter plot of baseline volumetric percentage of infection. Figure 2a demonstrates the decision-tree algorithm developed to classify COVID-19 pneumonia patients into common or severe group. Figure $2 \mathrm{~b}$ compared baseline volumetric

that the extent of abnormalities on CT increased in the first week and reached to peak at approximately 10-14 days, then the lesions were gradually decreased [16, 17]. In our study, both volumetric percentage and density increased at advanced stage, representing virus spread through bronchioles and alveolus to adjacent parenchyma causing infection,
B

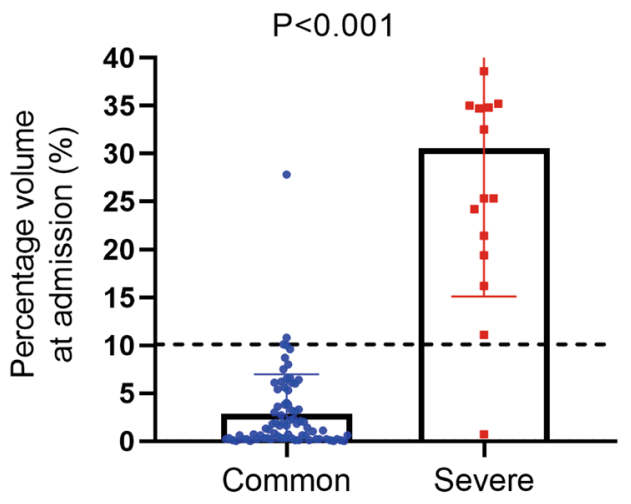

percentage between common and severe patients. The dotted line in black in Fig. 2b represents the cut-off value calculated by decisiontree. PPV positive predictive value; NPV negative predictive value

and consolidation replaced GGO, respectively. The peak time was earlier and the plateau was shorter in severe group. In the final stage, decreasing density meant that consolidation was absorbed and part of the consolidation turned into GGO (Fig. 4). However, different from the two previous studies, we divided COVID-19 pneumonia patients into
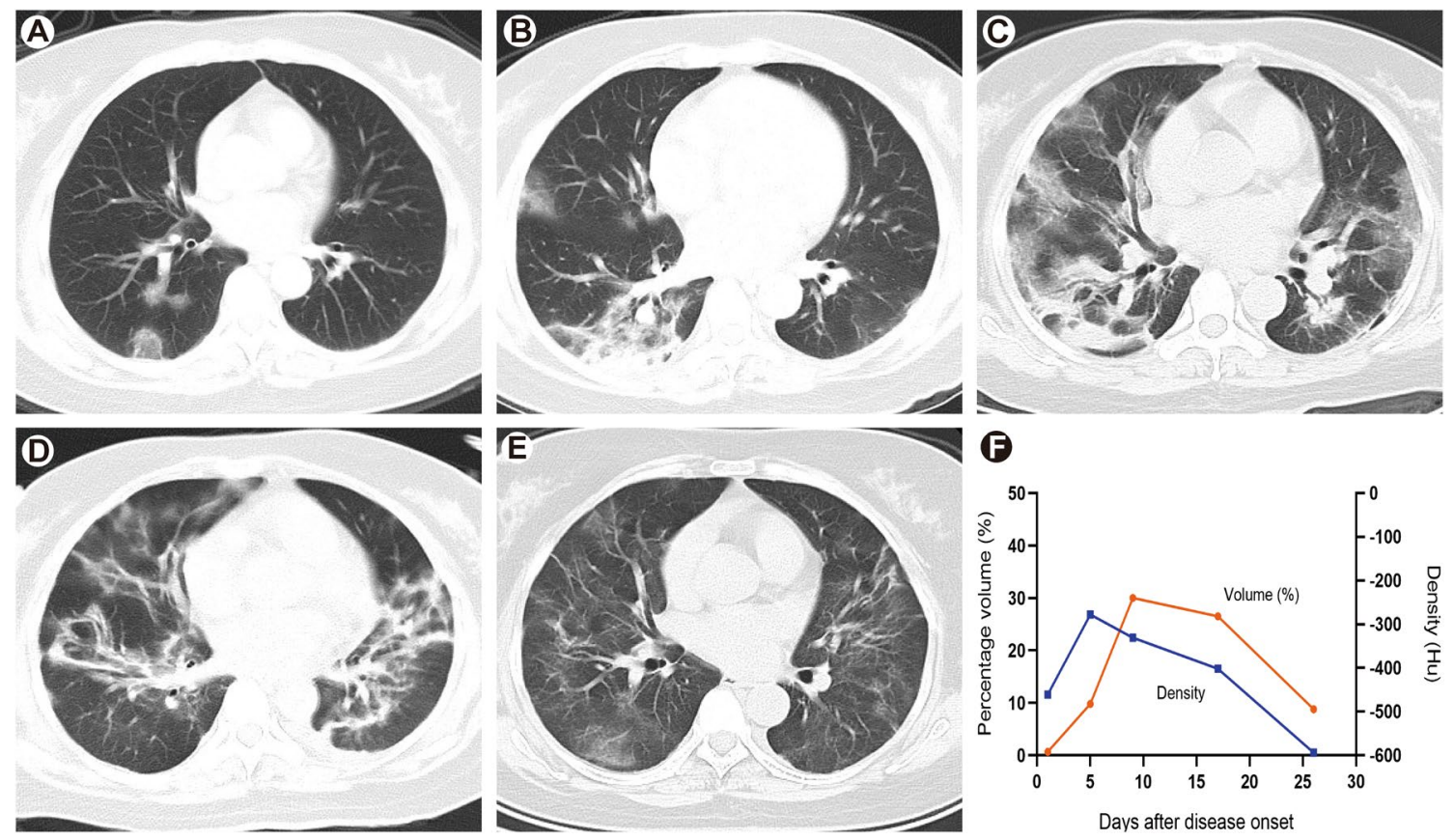

Fig. 4 Serial chest CT scans from a 51-year-old woman diagnosed as severe at admission. a Day 1 after disease onset: focal ground-glass opacities affected the left and right lower lobe. $\mathrm{SaO}_{2} 99 \%$. b Day 5: the main lesion located at the right lower lobe aggravated rapidly, with both extent and density increased. $\mathrm{SaO} 2$ 99\%. c Day 9: enlarged area of infection with bilateral, multifocal ground-glass opacities and consolidations. $\mathrm{SaO}_{2}$ decreased to $80 \%$ and patient was diag- nosed as severe. d Day 17: consolidations were absorbed and partially dissipated into ground-glass opacities. $\mathrm{SaO}_{2}$ returned to normal level (98\%) and patient's symptoms significantly relieved. e Day 26: infected areas continued to be absorbed, leaving extensive groundglass opacities. Patient discharged 2 days later. $\mathbf{f}$ Timeline demonstrates the dynamic change of volumetric percentage and density of this patient 
common and severe group based on current clinical criteria, and analyzed and compared quantifiable radiological data between the two groups, which made our data more accurate and reproducible.

During the absorption period, we noticed that while the density of infected regions decreased gradually, the total percentage of infection in some patients did not reduce or even continued to increase (especially in severe patients), but their symptoms relieved and $\mathrm{SaO}_{2}$ recovered to normal level (Fig. 5). This discrepancy may be explained as during the absorption of the main lesion, it dissipated into extensive range of GGO and caused expansion of infected region, which were identified by the software as new lesions. Thus, it indicates that severe patients may have longer absorption period and at this stage, density may play a more important role than volume in reflecting clinical course. These cases underline the necessity to analyze both infected volume and density (especially the main lesion), new and old lesions comprehensively.

In our study, the dynamic change of volumetric percentage and density was consistent with the clinical course of COVID-19 pneumonia. Patients who recovered and discharged from hospital had decreased volumetric percentage and density. Among those patients who aggravated from common to severe by clinical criteria, all of them also reached to the interquartile range of the severe degree, and the interval between diagnosis of severe by clinical parameters and by volumetric analysis was within 3 days in $73 \%$ patients. This consistency might be interpreted as the alveolar consolidation caused by inflammation, which directly affected the normal ventilation/perfusion (V/Q) homeostasis and thus compromised patients' pulmonary function, and this abnormality could be sensitively detected by chest CT. Thus, quantitative analysis of volume and density based on CT is correlated with respiratory function and can accurately reflect patients' clinical course [21].

Common patients had a relatively mild clinical course and better outcomes. However, most severe cases required mechanical ventilation and had a high risk of mortality. To make early classification of patients with COVID-19 pneumonia and tailor treatment according to patient's risk, we analyzed the baseline data of the two groups. Compared with common patients, severe patients were older and were more likely to have co-existing diseases, which was coincided with previous studies suggesting age and comorbidity might be risk factors for poor outcomes [6, 11]. In addition, compromised lung function at admission was also associated with poor prognosis as reported, and severe patients were more likely to experience acute respiratory distress syndrome (ARDS) [6, 7]. In our study, patients in severe group had a significantly higher total percentage of infection at the time of admission. Therefore, we hypothesized that higher volumetric percentage of infection at admission was associated with ARDS and was a risk factor for poor prognosis. To testify our hypothesis and provide practical implementation on clinical and radiologic classification, we used a decision-tree
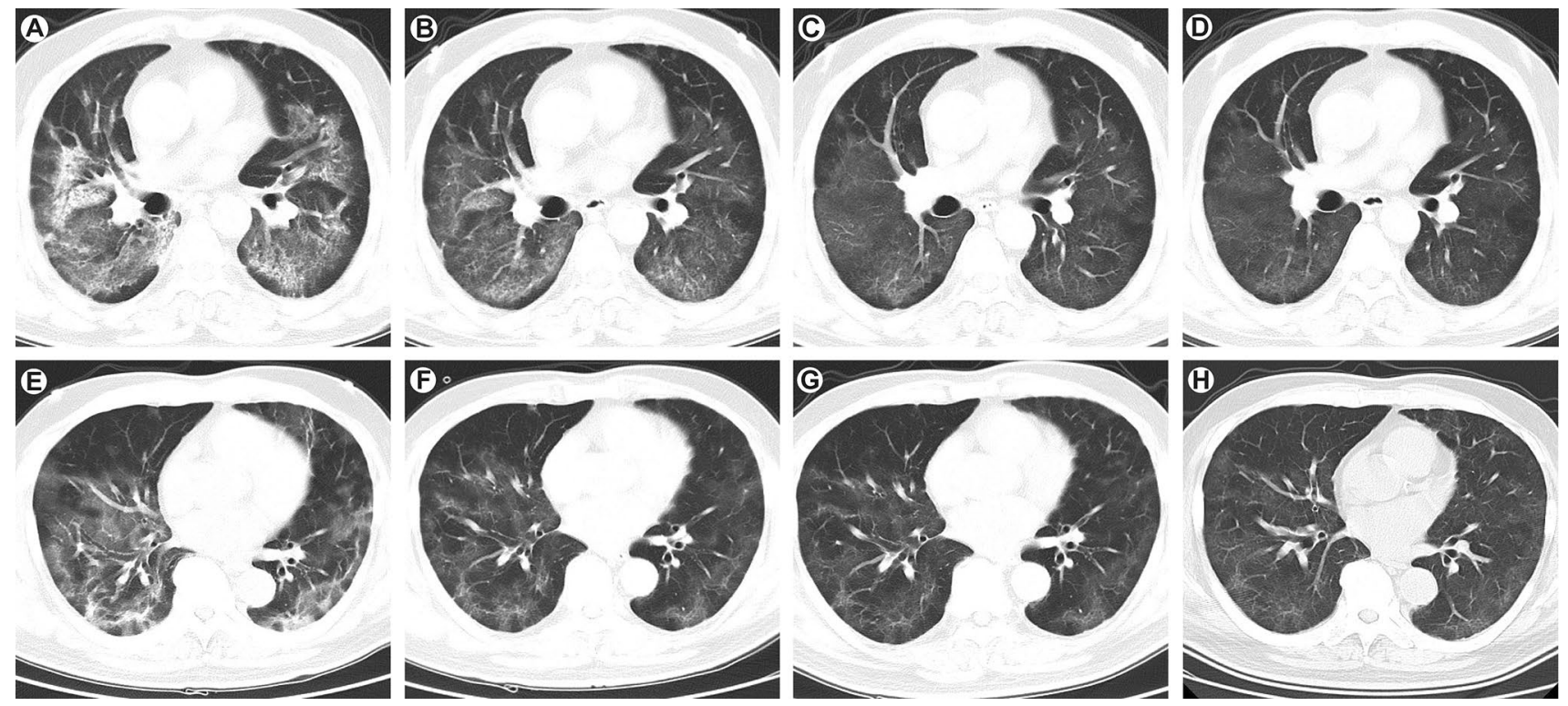

Fig. 5 Two cases in which volumetric percentage was inconsistency with density at absorption stage. a-d A 53-year-old man who was diagnosed as common to severe. Volumetric percentage reached to peak at day 20 and then absorbed. Although extent of infection did not decrease, but density decreased representing lesions were dis- sipating, and patient was recovering. e Another 65-year-old patient showed the same discrepancy during absorption period. Window level and window width of CT images were the same for each patient, respectively 
algorithm to determine the cut-off value of volumetric percentage of the common and severe degree. Although only one variable was used as a classifier, both sensitivity and specificity were within acceptable ranges, suggesting that this variable is efficient in classification.

This study has some limitations that should be considered. First, mild and critically ill patients were not included in our study. But given that mild patients had relatively better outcomes and critically ill patients often progressed to multiple organ failure, we believed chest CT had a low value in predicting prognosis in this subgroup of patients. Second, different radiological features (e.g., ground-glass opacities, reticulation, consolidation) were observed at different stage of clinical course, so our research could be further improved by analyzing and quantifying these specific features. Third, only seventeen patients were defined as severe in our cohort, which limited the ability to draw meaningful conclusions relative to the general population. Thus, further large-scale studies are needed to improve and validate the efficiency of the decision-tree in accurate classification.

In conclusion, patients with COVID-19 pneumonia diagnosed as the severe type by clinical parameters had significantly higher range of volumetric percentage of infection compared with common patients, and a cut-off value of volumetric percentage of $10.10 \%$ can accurately classify common and severe patients. Our research confirms the accuracy and utility of quantitative CT assessment on the diagnosis of COVID-19 pneumonia and provides a platform for further studies to complete the classification criteria for this novel disease.

Acknowledgements We thank all the staff in our department for their hard work on this manuscript and unselfish care for the enrolled patients. And we want to express our gratitude to Chuang Sun for statistical analysis

Authors contributions Conceptualization: BX; methodology: CY; data collection: GC and FL; data analysis and interpretation: $\mathrm{CY}$ and $\mathrm{JL}$; writing-original draft preparation: $\mathrm{CY}$ and $\mathrm{SH}$; writing review and editing: JL.

Funding Bin Xiong has received funding by National Natural Science Foundation of China (81873917).

Availability of data and material The datasets used and analyzed during the current study are available from the corresponding author on reasonable request.

Code availability Statistical analyses were processed using SPSS (version 25.0, USA).

\section{Declarations}

Conflicts of interest The authors declare no relationships with any companies, whose products or services may be related to the subject matter of the article.
Ethics approval The study protocol was complied with the principles of the Declaration of Helsinki and was approved by the institutional review board of the Union Hospital, Tongji Medical college, Huazhong University of Science and Technology.

Consent to participate Written informed consent was waived due to the retrospective nature of the study.

Consent for publication Not applicable.

\section{References}

1. Chen N, Zhou M, Dong X, et al. Epidemiological and clinical characteristics of 99 cases of 2019 novel coronavirus pneumonia in Wuhan, China: a descriptive study. Lancet. 2020;395:507-13.

2. Huang C, Wang Y, Li X, et al. Clinical features of patients infected with 2019 novel coronavirus in Wuhan, China. Lancet. 2020;395:497-506.

3. Zhu N, Zhang D, Wang W, et al. A novel coronavirus from patients with pneumonia in China, 2019. N Engl J Med. 2020;382:727-33.

4. Lu R, Zhao X, Li J, et al. Genomic characterisation and epidemiology of 2019 novel coronavirus: implications for virus origins and receptor binding. Lancet. 2020;395:565-74.

5. Xu X, Chen P, Wang J, et al. Evolution of the novel coronavirus from the ongoing Wuhan outbreak and modeling of its spike protein for risk of human transmission. Sci China Life Sci. 2020;63:457-60.

6. Yang $\mathrm{X}, \mathrm{Yu} \mathrm{Y,Xu} \mathrm{J,} \mathrm{et} \mathrm{al.} \mathrm{Clinical} \mathrm{course} \mathrm{and} \mathrm{outcomes} \mathrm{of}$ critically ill patients with SARS-CoV-2 pneumonia in Wuhan, China: a single-centered, retrospective, observational study. Lancet Respir Med. 2020. https://doi.org/10.1016/s2213-2600(20) 30079-5.

7. Guan WJ, Ni ZY, Hu Y, et al. Clinical characteristics of coronavirus disease 2019 in China. N Engl J Med. 2020. https://doi.org/ 10.1056/NEJMoa2002032.

8. General Office of National Health Committee (2020) Notice on the issuance of a program for the diagnosis and treatment of novel coronavirus infected pneumonia, 7th edn. http://www.nhcgoven/ yzygj/s7653p/202003/46c9294a7dfe4cef80dc7f5912eb1989shtml

9. Chassagnon G, Vakalopoulou M, Paragios N, Revel MP. Artificial intelligence applications for thoracic imaging. Eur J Radiol. 2020;123:108774.

10. Sul B, Flors L, Cassani J, et al. Volumetric characteristics of idiopathic pulmonary fibrosis lungs: computational analyses of highresolution computed tomography images of lung lobes. Respir Res. 2019;20:216.

11. Wang D, Hu B, Hu C, et al. Clinical characteristics of 138 hospitalized patients with 2019 novel coronavirus-infected pneumonia in Wuhan. China: Jama; 2020. https://doi.org/10.1001/jama.2020. 1585.

12. Bernheim A, Mei X, Huang $M$, et al. Chest ct findings in coronavirus disease-19 (COVID-19): relationship to duration of infection. Radiology. 2020. https://doi.org/10.1148/radiol.2020200463: 200463.

13. Pan $\mathrm{Y}$, Guan $\mathrm{H}, \mathrm{Zhou} \mathrm{S}$, et al. Initial $\mathrm{CT}$ findings and temporal changes in patients with the novel coronavirus pneumonia (2019nCoV): a study of 63 patients in Wuhan. China: Eur Radiol; 2020. https://doi.org/10.1007/s00330-020-06731-x.

14. Ai T, Yang Z, Hou H, et al. Correlation of chest CT and RT-PCR testing in coronavirus disease 2019 (COVID-19) in China: a report of 1014 Cases. Radiology. 2020. https://doi.org/10.1148/radiol. 2020200642:200642. 
15. $\mathrm{Li} \mathrm{K}, \mathrm{Wu} \mathrm{J}, \mathrm{Wu} \mathrm{F}$, et al. The clinical and chest $\mathrm{CT}$ features associated with severe and critical covid-19 pneumonia. Invest Radiol. 2020. https://doi.org/10.1097/rli.0000000000000672.

16. Pan F, Ye T, Sun P, et al. Time course of lung changes on chest ct during recovery from 2019 novel coronavirus (COVID-19) pneumonia. Radiology. 2020. https://doi.org/10.1148/radiol.20202 00370:200370

17. Shi H, Han X, Jiang N, et al. Radiological findings from 81 patients with COVID-19 pneumonia in Wuhan, China: a descriptive study. Lancet Infect Dis. 2020. https://doi.org/10.1016/s14733099(20)30086-4.

18. Grodecki K, Lin A, Cadet S, et al. Quantitative burden of COVID19 pneumonia on chest CT predicts adverse outcomes: a posthoc analysis of a Prospective International Registry. Radiology. 2020;2:e200389.

19. Huang L, Han R, Ai T, et al. Serial quantitative chest CT assessment of COVID-19: a deep learning approach. Radiology. 2020;2:e200075
20. Lanza E, Muglia R, Bolengo I, et al. Quantitative chest CT analysis in COVID-19 to predict the need for oxygenation support and intubation. Eur Radiol. 2020;30:6770-8.

21. Fernandez-Rodriguez L, Torres I, Romera D, et al. Prediction of postoperative lung function after major lung resection for lung cancer using volumetric computed tomography. J Thorac Cardiovasc Surg. 2018;156:2297-308.

Publisher's Note Springer Nature remains neutral with regard to jurisdictional claims in published maps and institutional affiliations. 